\title{
Aplicación de los métodos numéricos en la enseñanza superior
}

\author{
Application of numerical methods in higher education
}

\author{
Métodos numéricos
}

\author{
Robards Javier Lima Pisco.(1) \\ Julio Alberto Cedeño Ferrin.(2) \\ Migel Angel Padilla Orlando.(3)
}

(1) Universidad Estatal del Sur de Manabí, Jipijapa, Ecuador. email: robards.lima@ unesum.edu.ec

(2) Universidad Estatal del Sur de Manabí, Jipijapa, Ecuador. email: julio.cedeno@unesum.edu.ec

(3) Universidad Estatal del Sur de Manabí, Jipijapa, Ecuador. email:, miguel.padilla@ unesum.edu.ec

Contacto: julio.cedeno@unesum.edu.ec

Recibido: 17-04-2020

Aprobado: 31-05-2020

\section{Resumen}

En el presente trabajo se analiza las incidencias de las tecnologías de la información y la comunicación (TIC) en el proceso enseñanzaaprendizaje de métodos numéricos en la formación de los estudiantes de Ingeniería en Tecnologías de la Información, Universidad Estatal del Sur de Manabí. El propósito de este trabajo es identificar el uso de las TIC, y su incidencia en el proceso enseñanza-aprendizaje de los métodos numéricos para encontrar raíces en funciones polinómicas en la formación de los futuros profesionales, así como determinar si las herramientas utilizadas por el docente son las más adecuadas. La metodologia utilizada aborda desde un paradigma de investigación mixta pues se examinan aspectos de visión cuantitativa y la posibilidad de incidir en políticas con visión cualitativas. El diseño de este trabajo es no experimental, ya que trabaja con estudiantes matriculados en la asignatura de métodos numéricos. Dentro de los resultados obtenidos se evidencio que las TIC mejoran el proceso enseñanza- aprendizaje de los métodos numéricos para determinar los distintos métodos de cálculo. Se concluyó seguir investigando sobre otra herramienta a ser utilizadas.

Palabras clave: comunicación, cuantitativa, cualitativas, incidencias

\footnotetext{
Abstract

This paper analyzes the incidences of information and communication technologies (ICT) in the teaching-learning process of numerical methods in the training of students of Engineering in Information Technology, Universidad Estatal del Sur de Manabí. The purpose of this work is to identify the use of ICT, and its impact on the
}

teaching-learning process of numerical methods to find roots in polynomial functions in the training of future professionals, as well as to determine if the tools used by the teacher are the most suitable. The methodology used approaches from a mixed research paradigm since aspects of quantitative vision and the possibility of influencing policies with a qualitative vision are examined. The design of this work is non-experimental, since it works with students enrolled in the subject of numerical methods. Among the results obtained, it was evidenced that ICT improve the teaching-learning process of numerical methods to determine the different calculation methods. It was concluded to continue investigating another tool to be used.

Key words: communication, quantitative, qualitative, incidents

\section{Introducción}

La asignatura Método Numéricos, es una materia que está dentro del pensum académico de la nueva carrera Ingeniería en Tecnologías de la Información (TI), rediseñada en la facultad de ciencias técnicas, de la Universidad Estatal de Sur de Manabí, dictada en cuarto semestre, los estudiantes que se matriculan en la carrera (TI) para ver esta asignatura deben ver aprobado Cálculo Diferencial e Integral.

Molina (2005), afirma que los temas tratados en esta temática, tienen el propósito de dar solución a ecuaciones con una solo variable, o para hallar raíces, entre los cuales se han seleccionado, método de Bisección, método de NewtonRaphson, Método de la Secante, Regla Falsa. Luego de haber realizado un análisis con los estudiantes se observó en su mayoría 
desconocimiento y dificultad en el manejo de estos métodos.

Granados (2015), en un estudio analizó la incidencia de las Tecnologías de la Información y la Comunicación (TIC), en el proceso de enseñanza de los métodos numéricos en la formación de los estudiantes de ingeniería de sistemas de la corporación Universitaria de la Costa (CUC), señala que el propósito de la investigación fue identificar cuáles son las herramientas tecnológicas más usadas por los docentes.

Los autores de este artículo consideran que las TIC tienen un impacto favorable en el proceso enseñanza-aprendizaje de la asignatura métodos numéricos en los temas de raíces en funciones polinómicas en la formación de los estudiantes de (TI) de la Universidad Estatal del Sur de Manabí (UNESUM)

El propósito de este trabajo es identificar el uso de las TIC, y su incidencia en el proceso enseñanzaaprendizaje de los métodos numéricos para encontrar raíces en funciones polinómicas en la formación de los futuros profesionales, así como determinar si las herramientas utilizadas por el docente son las más adecuadas.

\section{Materiales y métodos}

La investigación fue no experimental, porque en ella no se realizó la manipulación deliberada de variables y se trabajó con un grupo específico de estudiantes matriculados en la carrera Tecnologías de la Información Facultad de Ciencias Técnicas de la "UNESUM", Ecuador. A su vez la investigación fue de tipo descriptiva, ya que en ella

$$
\begin{aligned}
& \mathrm{n}=\frac{N}{1+e^{2 * 87}}=\frac{87}{\left(1+\left((0,09)^{2} * 87\right)\right.}=\frac{87}{(1+(0,0081 * 87)}=\frac{87}{(1+(0,7047)}= \\
& \frac{87}{(1,7047)}=51
\end{aligned}
$$

$\mathrm{n}=51$ estudiantes estudiante carrera (TI) a encuestar.

Después de llevar a cabo la encuesta a los estudiantes se procedió a tabular los datos para hacer un análisis de los mismos, se hizo uso del Excel para poder realizar las tablas y las gráficas. Con estos resultados se procedió a realizar el análisis de los mismos, para luego sacar las conclusiones.

\section{Las TIC en la enseñanza superior}

se relataron las características fundamentales de las TIC usadas en la enseñanza de los métodos numéricos para hallar raíces.

El estudio se llevó a cabo en los cuartos semestres de la carrera tecnologías de la información en la facultad de ciencias técnicas sección matutina y vespertina en el periodo académico mayo septiembre 2019.

Durante el desarrollo de la investigación se realizaron encuestas a estudiantes que cursaban del cuarto nivel la carrera (TI) en la asignatura de Métodos Numéricos También se trabajó con los dos profesores de la asignatura de métodos numéricos.

Teniendo en cuenta el tipo de proyecto realizado, para determinar el tamaño de la muestra, se hace uso de la siguiente formula estadística:

$$
\mathrm{n}=\frac{N}{1+e^{2} * 87}
$$

Donde:

$\mathrm{n}=$ Representa el tamaño de la muestra sobre la cual se realizó la encuesta.

$\mathrm{N}=$ Representa la población objetivo.

$\mathrm{e}=$ Representa el porcentaje de error

Para este proyecto, $\mathrm{N}$ está dado por 87 personas, el error (e) en este cálculo está estimado en el 9 $\%$, por lo cual el tamaño de la muestra es el siguiente:

Los docentes en la actualidad utilizan las TIC en los procesos de enseñanza- aprendizaje con un análisis exhaustivo relacionado a la globalización y del entorno cada vez más cambiante en la que se mueven las instituciones de la educación superior (Martínez, 2011).El uso de las TIC supone para los docentes supone un desafío en la utilización de medios más sofisticados que se emplean en la enseñanza.

De la misma manera lo indica Schiavo, (2007), el principal uso de las TIC es utilizarla como una herramienta que acelera procesos y por tal motivo 
disminuir el tiempo de dedicación de los mismos. Otro uso de gran importancia es que sirve para organizar diversas actividades e interactuar en el espacio-tiempo que estas tecnologías generan.

En definitiva, las TIC, con toda la gama de herramientas de hardware y software que contiene, facilitan la creación de ambiente de aprendizaje enriquecidos, con excelentes resultados en el desarrollo de las habilidades cognitivas (Eduteca, 2007)

Algunos recursos considerados TIC que pueden ser utilizados por los docentes dentro del apoyo en la formación de los estudiantes de acuerdo a (Arrieta, Flórez y Martínez, 2010)

Videoconferencia, buscadores, software especializado (procesadores de texto, hojas de cálculo, bases de datos, programas de presentaciones gráficas, etc.)

Espacios virtuales de comunicación (foros, debates,...), correo electrónico, chats y mensajería instantánea, materiales didácticos multimedia (en línea o local), listas de discusión/distribución

Hardware (impresora, escáner, cámara digital, etc.)

En el proceso de enseñanza-aprendizaje de la actualidad, se debe tener en cuenta que este tiene unas características importantes: el modelo pedagógico tendrá una didáctica fundamentada en la teoría de la inteligencias múltiples, una metodología de aprendizaje de carácter constructivista, la virtualidad será el eje motor de los procesos de aprendizaje y la creatividad incorporando un mayor uso del internet y de las TIC. (Nieto, 2009).

\section{Enseñanza de métodos numéricos mediada por TIC}

Los Métodos Numéricos no pueden dejar la utilización de las herramientas que la tecnología nos brinda, haciéndose necesario la incorporando nuevas técnicas o herramientas por medio de la cual el proceso de enseñanza aprendizaje mejore.

En el caso de los Métodos numéricos Chapra y Canales, (2007), describen en su libro de Métodos numéricos aplicado a la ingeniería, muestran como antes de la aparición del computador y de su uso en los métodos numéricos la mayor parte de la energía se gastaba en la técnica de solución, en lugar de la definición e interpretación del problema.

En este sentido el uso de las páginas web que contengan temas relacionados con los Métodos Numéricos también brindan un gran apoyo, ya que en ellas no se tienen en cuenta el tiempo, ni el espacio, así el estudiante puede acceder a ellas sin necesidad de que el docente esté presente, y sin importar el sitio donde se encuentre, lo importante es tener el acceso a la red de redes, Internet (Granados, 2009).

\section{Resultados}

Este trabajo muestra las acciones emprendidas en este estudio, los participantes en la investigación fueron 51 estudiantes. En el instrumento aplicado, los resultados reflejan lo siguiente en lo relativo a la pregunta: ¿Considera que a través del uso de las TIC en la asignatura de Métodos Numéricos lograrías desarrollar competencias científicas y un mejor aprendizaje? (si) (no), se observa en la tabla 1.

\begin{tabular}{|l|l|l|}
\hline Alternativas & Numero de encuestados & Porcentaje \\
\hline si & 45 & $88 \%$ \\
\hline no & 6 & $12 \%$ \\
\hline total & 51 & $100 \%$ \\
\hline
\end{tabular}

Tabla 1. Uso de las TIC en la asignatura de Métodos Numéricos lograrías desarrollar competencias científicas y un mejor aprendizaje

Los resultados refleja en el gráfico Nro. 1, que más del $88 \%$ de los estudiantes consideran que el uso de diapositivas o presentaciones en PowerPoint, videos o software educativos incrementan la madurez cognitiva en el estudiante y fortalecen su aprendizaje en el aula. 


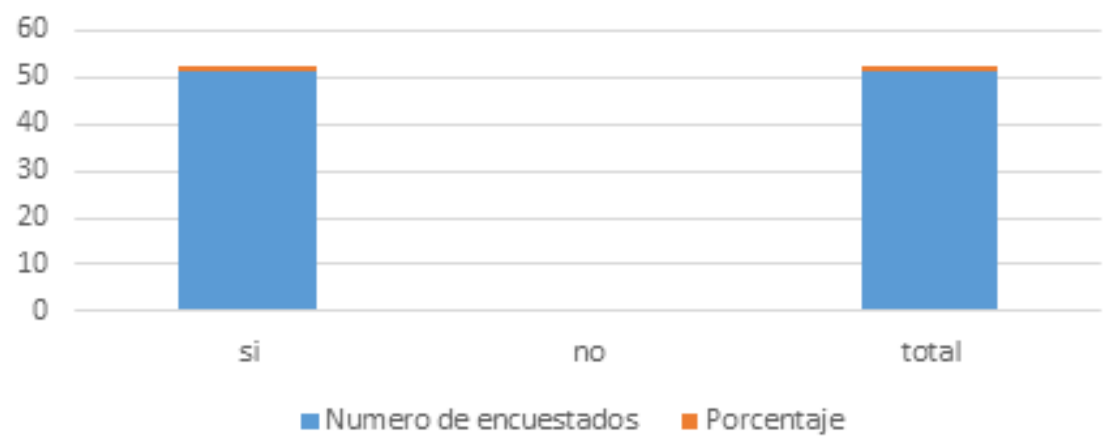

Gráfico 1. Uso de diapositivas, videos y software educativo

En cuanto a la pregunta relacionada así se incrementa la utilización de diapositivas, videos y software educativos la madures cognitiva en el aprendizaje de Métodos Numéricos, los resultados se observan en la tabla 2 .

\begin{tabular}{|l|l|l|}
\hline Alternativas & Numero de encuestados & Porcentaje \\
\hline $\mathrm{Si}$ & 48 & $94 \%$ \\
\hline $\mathrm{No}$ & 3 & $6 \%$ \\
\hline total & 51 & $100 \%$ \\
\hline
\end{tabular}

Tabla 2. Consideras que se debe incrementar la utilización de diapositivas, videos y software educativos en el aprendizaje.

Al realizar la pregunta sobre el uso del Internet como herramienta pedagógica que fortalece el aprendizaje significativo de contenidos, todos los estudiantes que formaron parte del estudio plantean que es vital el uso de estas herramientas, gráfico 2.

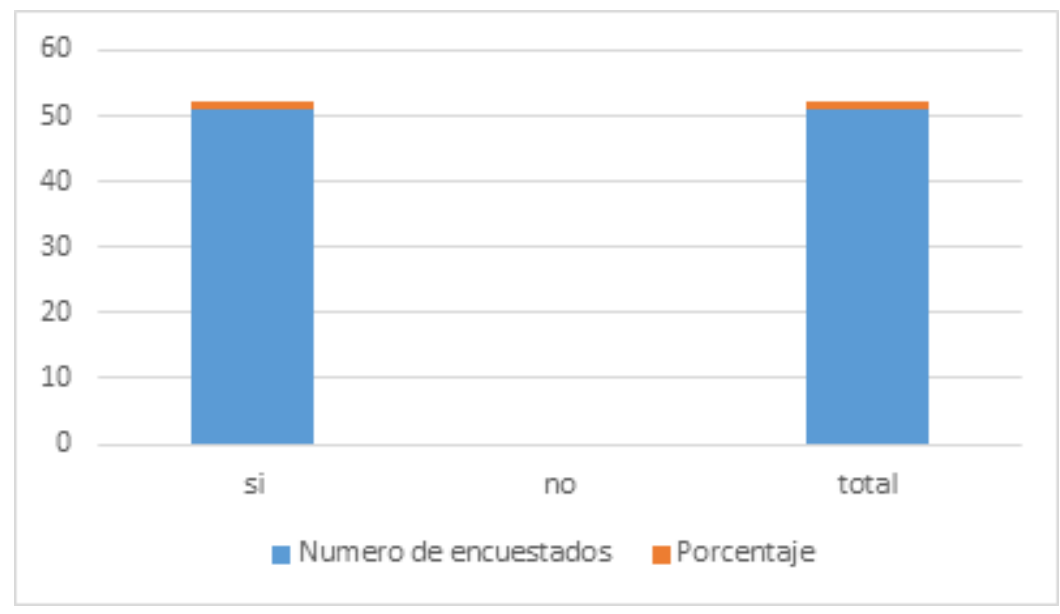

Gráfico 2. Uso de Internet como herramienta pedagógica

En lo concerniente al aprendizaje significativo de contenidos y la actitud hacia estrategias de aprendizaje significativo en la enseñanza de Métodos Numéricos cuando se utiliza Internet en el aula como herramienta pedagógica, el $78 \%$

\begin{tabular}{|l|l|l|}
\hline Alternativas & Numero de encuestados & Porcentaje \\
\hline si & 40 & $78 \%$ \\
\hline no & 11 & $22 \%$ \\
\hline total & 51 & $100 \%$ \\
\hline
\end{tabular}

Tabla 3. Consideras que se debe incrementar la utilización de estrategias de aprendizaje significativo en la enseñanza de Métodos Numéricos cuando se utiliza Internet. plantea que sí, mientras que el $22 \%$, indica que no, se expresan los resultados en la tabla 3 . 
La información presenta que a pesar de que la mayoría se sienten satisfechos con el acceso al uso de las TIC, cabe anotar que algunos estudiantes manifestaban su inconformidad por no tener salas disponibles a ciertas horas, pero otros anotaban que con el Wifi no era necesario estar en las salas, gráfico 3

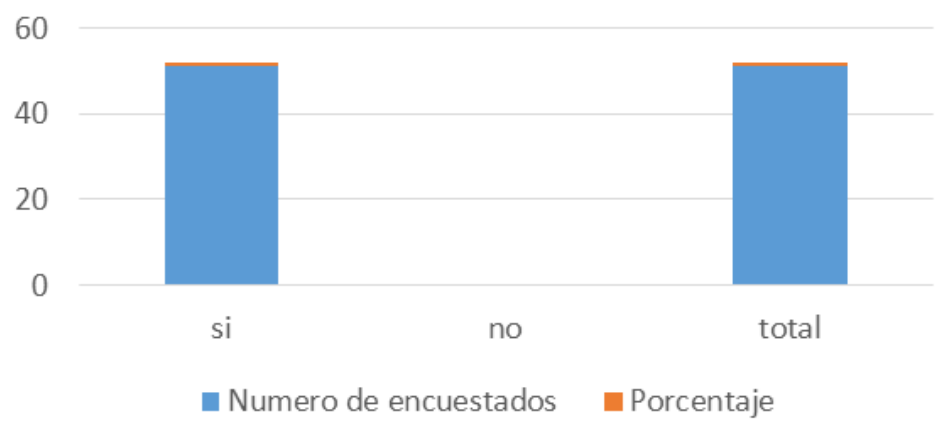

Gáfico 3. Uso de las TIC en la docencia.

En lo relacionado al uso de la plataforma Moodle así ayuda esta herramientas a la mejor comprensión de los Métodos Numéricos para hallar raíces, el

\begin{tabular}{|l|l|l|}
\hline Alternativas & Numero de encuestados & Porcentaje \\
\hline si & 51 & 100 \\
\hline no & 0 & 0 \\
\hline total & 51 & $100 \%$ \\
\hline
\end{tabular}

Tabla 4. Plataforma Moodle y su uso en la docencia

Todos los estudiantes consideraron que el Moodle ayuda a mejorar la comprensión de los métodos numéricos, además que le sirve al docente para subir en ella el material de los temas tratados e indicar los trabajos independientes que deben realizar a los estudiantes y se fortalece el contrustivismo, gráfico 4.

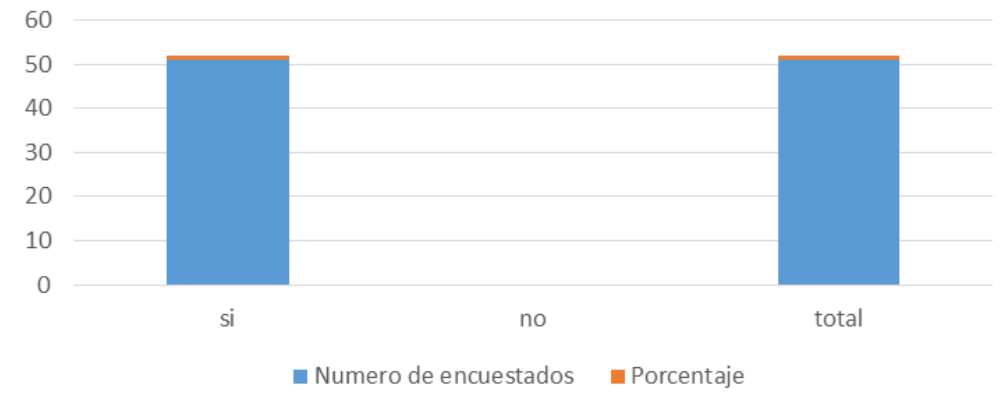

Gráfico 4. Uso de la plataforma Moodle en la docencia.

Los estudiantes consideraron que la página Web ayuda a mejorar la comprensión de los métodos numéricos, ya que en ella encuentran los programas, algoritmos y explicación de los métodos, (Gráfico 5).

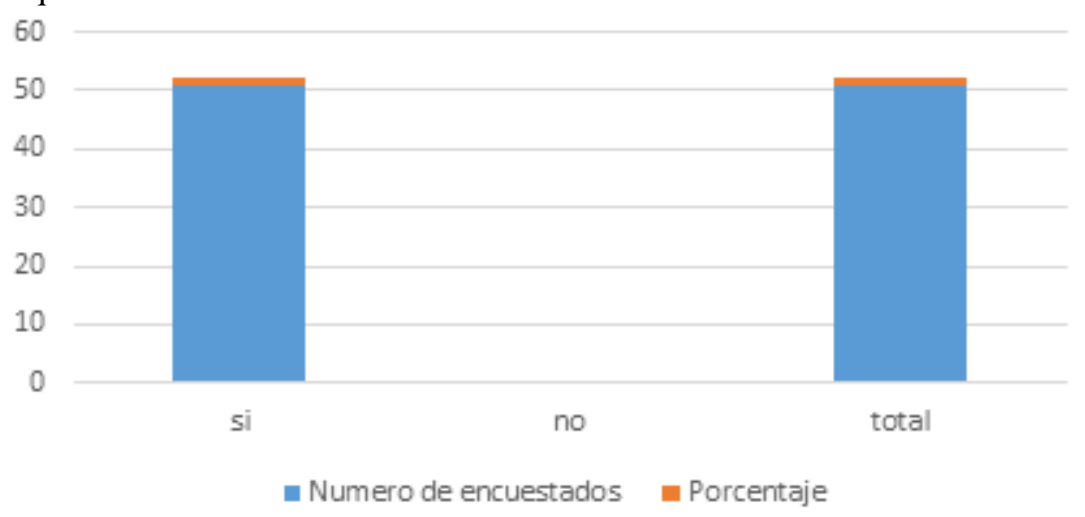


Gráfico 5. Uso de las páginas web para mejorar la comprensión de los métodos numéricos.

\section{Discusión.}

En esta experiencia se concuerda con la investigación emprendida por Granado en el (2015) que asevera que las TIC mejoran el proceso de enseñanza de los métodos numéricos para hallar raíces,en la formación de los estudiantes de Ingeniería de Sistemas de la en la Corporación Universitaria de la Costa, en Colombia.

También se concuerda con otra investigación que señala, la diversidad en los métodos, es de mucha importancia, cuando se tratan de resolver variadas situaciones de tipo problemática o algún problema específico que enfrente el profesional de ingeniería; porque con ello se permite además, elegir cual o en qué casos se puede aplicar un determinado procedimiento, que conocimientos previos se tienen para su utilización, cual es u eficiencia, etcétera (Molina, 2005).Siguiendo lo planteado por Arias, Sandia, \& Mora (2012), argumentan en su estudio que el el empleo de las herramientas tecnológicas web, así como la plataforma Moodle en la didáctica interactiva a distancia, son eficientes, efectivas y eficaces, de acuerdo al cómo el profesor las utilice en beneficio de fortalecer el aprendizaje, en esta investigación se concuerda con estos autores.

\section{Conclusiones}

Se concluye en que las TIC mejoran el proceso de enseñanza de los métodos numéricos para hallar raíces en las funciones polinómicas en la formación de los estudiantes de Ingeniería en Tecnologías de la Información de la Universidad Estatal del Sur de Manabí, ya que teniendo en cuenta lo manifestado por ellos en las encuestas y en las observaciones realizadas sobre las TIC declaraban que estas herramientas le serían de mucha ayuda en su proceso y que le posibilitan afianzar lo aprendido.

Se encontró que las TIC usadas por los docentes en la enseñanza de los métodos numéricos para hallar raíces en funciones polinómicas en la formación de los futuros profesionales de Ingeniería en Tecnologías de la Información son: el internet, las páginas web, los blogs, las bases de datos especializadas y la plataforma Moodle como herramientas.

Los estudiantes realizan programas, en software ya creado para verificar sus resultados, usan videos de internet para aclarar las dudas que tienen, envían y consultan información atreves del Moodle. Todo esto hace que el uso de las TIC incidan en el proceso de enseñanza de los métodos numéricos, para hallar raíces en funciones polinómicas.

\section{Bibliografías.}

Arias , M., Sandia, B., Mora, E. (2012). La didáctica y las herramientas tecnológicas web en la educación interactiva a distancia Revista Educere, 16, (53), 2136.Recuperado de https://www.redalyc.org/pdf/356/3562353 8004.pdf

Arrieta, R., Flores, M., \& Martínez, O. (2010). Articulación Pedagogía - Tecnología: Un Medio Para Mejorar Las Competencias Del Lenguaje Y La Comunicación. Barranquilla, Educosta. 159 pág.

Burden, R. (2001). Análisis Numérico. México, Thompson Learning. Séptima edición. Pag.839.

Castro, W. \& Godino J. (2011). Métodos Mixtos De Investigación En Las Contribuciones A Los Simposios De La Seiem (1997-2010), págs. 99-116. Recuperado de: http://dialnet.unirioja.es/servlet/articulo?co $\underline{\operatorname{dig}}=3731076$

Chapra, S., \& Canale, R. (2007). Métodos Numéricos Para Ingenieros. México: Ed.McGraw Hill.

Eduteka (2007). El porqué de las TIC en la Educación. Recuperado de http://www.eduteka.org/PorQueTIC.php

Granados, A. (2009). Revista Inge-CUC. Métodos Numéricos y la Web, Pag 249-258. Barranquilla, Educosta.

Granados, (2015). Las TIC en la enseñanza de los métodos numéricos. Revista Sophia, 11, (2), 143-154. Recuperado de https://www.redalyc.org/pdf/4137/413740 778003.pdf

Martínez, O (2011).La Virtualidad: un reto para las Instituciones de Educación Superior presenciales. Barranquilla, Educosta.

Molina, F. (2005). Desarrollos alternativos en Raíces de Ecuaciones. PROSPECTIVA, vol. 3, (1), 38-44. Recuperado de https://www.redalyc.org/pdf/4962/496251 105008.pdf 
Nieto, M. (2009). Espacios educativos y docentes virtuales con las TIC. Recuperado de http://mnieto2009.lacoctelera.net/categoria /articulos-sobre-tics

Schiavo, E. (2007). Investigación científica y tecnológica en el campo de las TIC ¿Conocimientos técnicos, contextuales o transversales? Rev. Iberoam. Cienc. Tecnol. soc., 3, (9). Recuperado de http://www.scielo.org.ar/scielo.php?script $=$ sci arttext\&pid=S1850

Unesco. (2008) Estándares de competencia en TIC para docentes. Londres. Recuperado de

http://www.oei.es/tic/UNESCOEstandares Docentes.pdf 